\title{
Rosai-Dorfman disease in a 12-year-old Nigerian male
}

\author{
${ }^{1}$ Department of Paediatrics, Federal Medical Centre, Owerri, Nigeria \\ ${ }^{2}$ Department of Histopathology, Federal Medical Centre, Owerri, Nigeria
}

J C Elo-Ilo, ${ }^{1}$ MBBS, FWACP; K K Odinaka, ${ }^{1}$ MBBS, FWACP; C O Ukah, ${ }^{2}$ MBBS, FMC Path

Corresponding author: K K Odinaka (kellymed112@yahoo.com)

\begin{abstract}
Rosai-Dorfman disease is a rare disorder of systemic histioproliferation, first described in 1969 by Juan Rosai and Ronald Dorfman. It is characterised by massive lymphadenopathy, particularly of the head and neck region, and it is sometimes associated with extranodal involvement. This is the first case to be reported from our centre. We report a case of Rosai-Dorfman disease in a 12-year-old Nigerian boy who presented at the children's outpatient clinic of the Federal Medical Centre, Owerri, Imo State, Nigeria, with a 5-year history of bilateral neck swelling, a 2-year history of groin swelling and a recurrent fever of over a year's duration. An initial diagnosis of Hodgkin's lymphoma was made. An excisional biopsy of the cervical lymph node was undertaken and the histology showed massive histiocytosis with emperipolesis, which confirmed the diagnosis of Rosai-Dorfman disease.Rosai-Dorfman disease, although a rare clinical entity, should be suspected in patients presenting with massive lymphadenopathy located especially in the neck and groin regions.
\end{abstract}

S Afr J Child Health 2016;10(4):237-238. DOI:10.7196/SAJCH.2016.v10i4.1197

Rosai-Dorfman disease (RDD), otherwise known as sinus histiocytosis with massive lymphadenopathy (SHML), is a rare and benign histiocytic proliferative disorder of unknown aetiology. ${ }^{[1]}$ It was previously classified as histiocytosis X. Patients with the disease usually present with massive painless cervical lymphadenopathy and fever. About $25-40 \%$ of affected individuals present with extranodal manifestations, typically in the head and neck regions. The skin, soft tissue, upper respiratory tract, bone, eyelids, retro-orbital tissue and the kidneys may also be involved. ${ }^{[1,2]}$ RDD is most frequently seen in children and young adults, although it can occur in any age group. The disease is more common in males, with a male:female ratio of $2: 1$. It is also common in individuals of African descent and usually occurs in the first two decades of life. ${ }^{[3]}$ The clinical course of RDD is unpredictable, with episodes of exacerbation and remissions that can last many years. ${ }^{[2]}$ The disease is often self-limiting and therefore requires no treatment, with a very good outcome in the majority of cases. ${ }^{[2]}$ However, treatment may be required for patients with extranodal RDD involving vital organs such as orbits, or those with nodal disease, causing life-threatening complications. ${ }^{[2]}$ Various treatment options have been recommended if required and they include the use of corticosteroids, interferon, radiation, chemotherapy and surgery. ${ }^{[4,5]}$ We are reporting the case of RDD in a 12-year-old Nigerian male to highlight the need for physicians to evaluate children with massive lymphadenopathy for the likelihood of this rare disease entity.

\section{Case presentation}

A 12-year-old Nigerian male presented at the children's outpatient clinic of the Federal Medical Centre, Owerri, Nigeria, with a 5-year history of bilateral neck swelling, 2-year history of groin swelling and a 1-year recurrent fever. There was no history of cough, weight loss or drenching night sweats. At the onset of the illness, he was taken to a private hospital where he was placed on anti-tuberculosis (TB) drugs for 6 months, with no improvement. He also received a course of herbal concoctions, again with no improvement. The neck swelling continued to increase, necessitating self-referral to our facility for expert medical care. His past medical history was not significant except that he had been transfused once in a private hospital because of febrile illness when he was 6 years old. He is the third child in a family of seven children. There was no history of similar illness among his siblings. Physical examination revealed mild pallor, bilateral cervical lymphadenopathy measuring $8 \times 6 \mathrm{~cm}$ on the left and $6 \times 4 \mathrm{~cm}$ on the right. The nodes were multiple, firm and attached to neither superficial or deep tissues. He also had left inguinal lymphadenopathy measuring $8 \times 8 \mathrm{~cm}$, and $2 \times 2 \mathrm{~cm}$ on the right. There was no positive cough impulse. His weight was $70 \%$ of the expected weight without oedema, and his height was appropriate for his age. Other aspects on examination were essentially normal. A working diagnosis of Hodgkin's lymphoma with tuberculous lymphadenopathy as a differential diagnosis was made. The laboratory investigations showed a white blood cell count of $13.8 \times$ $10^{9} \mathrm{~L}$, neutrophils $70 \%$, lymphocytes $30 \%$, platelet count $188 \times 10^{9} \mathrm{~L}$, left shift of neutrophils, normocytic normochromic red blood cells and elevated erythrocyte sedimentation rate (ESR) $(100 \mathrm{~mm} / \mathrm{hr})$. Haemoglobin was $9.8 \mathrm{~g} / \mathrm{dL}$, HIV I and II screenings were negative and haemoglobin electrophoresis had a normal AA pattern. A Mantoux test was non-reactive $(0 \mathrm{~mm})$. A chest X-ray showed no evidence of enlarged lymph nodes and no focal lung lesions were seen. Blood films for detecting malaria parasites showed a ring form of Plasmodium falciparum. An abdominal ultrasound scan, random blood glucose tests, urinalysis and direct Coombs tests were all normal. The patient was commenced on oral antibiotics (cefuroxime) and antimalarial therpay (Coartem) based on local sensitivity patterns and malarial tests, pending the result of the excision biopsy of the lymph node.

The histology report showed a benign, non-neoplastic lesion characterised by expanded sinuses that were filled with enlarged histiocytes containing abundant cytoplasm and plasma cells. Within some of the enlarged histiocytes were intact lymphocytes (emperipolesis). A histological diagnosis of RDD was made. The parents were counselled on the benign nature of the disease and were scheduled for monthly follow-up visits. The size of the cervical lymph node was observed to be regressing by the third monthly visit. However, the parents defaulted on further follow-up visits despite numerous telephonic requests.

\section{Discussion}

The term RDD was first introduced into scientific literature in 1969 when Juan Rosai and Ronald Dorfman described four cases of a rare, idiopathic, non-neoplastic lymphoproliferative disorder. ${ }^{[6]}$ They described its clinico-pathological features in 1972. Typically, it is characterised by bilateral cervical lymphadenopathy with fever, leukocytosis, elevated ESR and hypergammaglobunaemia. ${ }^{[7]}$ Our 
patient had all the typical features of the disease except that we could not ascertain if he had hypergammaglobunaemia because we lacked the facilities to test for gammaglobunin. The extranodal manifestation of the disease has been documented in about $25-40 \%$ of affected individuals, with the most frequent sites being the head and neck regions..$^{[1,2]}$ The urogenital tract, breast, gastrointestinal tract, liver, pancreas and lungs have also been reported to be affected by the disease. ${ }^{[2]}$ Our patient did not have any extranodal disease.

Histologically, lymph nodes in this disease show pericapsular fibrosis and dilated sinuses, heavily infiltrated with large histiocytes, lymphocytes and plasma cells. The presence of emperipolesis, or the engulfment of lymphocytes and erythrocytes by histiocytes that express S-100, is considered diagnostic of RDD. ${ }^{[2]}$ In this case, the authors lacked the facilities for testing an immunohistochemical stain for S-100 positivity in histiocytes, and therefore it was not done on this patient's biopsy.

Although the aetiology of RDD is unknown, it is presumed to be due to an immune regulation disorder. Patients with clinically apparent immunological disorders have a higher mortality. There also appears to be a familial relationship with regard to RDD: two sets of twins, two other sets of siblings and four individuals of blood relatives were documented by Foucar ${ }^{[8]}$ in 1984 . RDD has also been presumed to be triggered by infections caused by viruses such as various herpes viruses, the Epstein-Barr virus, cytomegalovirus, Brucella and Klebsiella. ${ }^{[9,10]}$ However, there is presently no strong evidence for this. ${ }^{[2]}$ Stebbing et al. ${ }^{[1]}$ reported a possible association with sickle cell disease. The haemoglobin electrophoresis of our patient was AA. This implies that a person's genotype may not play a contributory role. However, our patient and the patient of Stebbing et al. ${ }^{[11]}$ had been transfused in the past. Could there be a possible association of RDD with blood transfusion? More research will be required to address this question. It is noteworthy that RDD has been reported following bone marrow transplants for precursor-B acute lymphoblastic leukaemia. ${ }^{[12]}$

As previously mentioned, the patient was lost to follow-up, probably because of emotional burn-out from repeated hospital visits and perceived slow response to treatment. Similar observations have been documented in parents of children with incurable chronic diseases in Nigeria. ${ }^{[13]}$

\section{Conclusion}

This case report highlights this rare disease entity in order to raise a high index of suspicion on diagnosis. Patients who present with lymphadenopathy and who do not respond to treatment with antibiotics or anti-TB treatment must be followed up. These patients may need a biopsy to exclude malignant diseases or other diseases such as RDD.

1. References Santra G, Das BK, Mandal B, Kundu SS, Bandopadhyay A. RosaiDorfman disease. Singapore Med J 2010;51(10):e173-e175.

2. Histiocyte Society. Rosai-Dorfman Disease. 2011. http://www.histiocytesociety. org.http://histiocytesociety.org/document.doc?id=54 (accessed 20 March 2016).

3. Lauwers GY, Perez-Atayde A, Dorfman RF, Rosai J. The digestive system manifestations of Rosai-Dorfman disease (sinus histiocytosis with massive lymphadenopathy): Review of 11 cases. Hum Pathol 2000;31(3):380-385. http:// dx.doi.org/10.1016/s0046-8177(00)80254-3

4. Horneff G, Jürgens H, Hort W, Karitzky D, Göbel U. Sinus histiocytosis with massive lymphadenopathy (Rosai-Dorfman disease): Response to methotrexate and mercaptopurine. Med Pediatr Oncol 1996;27(3):187-192. http://dx.doi. org/10.1002/(sici) 1096-911x(199609)27:3<187::aid-mpo10>3.0.co;2-d

5. Löhr HF, Gödderz W, Wölfe T, et al. Long-term survival in a patient with RosaiDorfman disease treated with interferon-alpha. Eur J Cancer 1995;31A(1314):2427-2428. http://dx.doi.org/10.1016/0959-8049(95)00375-4

6. Rosai J, Dorfman RF. Sinus histiocytosis with massive lymphadenopathy. A newly recognized benign clinicopathological entity. Arch Path 1969;87(1):6370 .

7. Foucar E, Rosai J, Dorfman RF. Sinus histiocytosis with massive lymphadenopathy (Rosai-Dorfman disease): Review of the entity. Semin Diagn Pathol 1990;7(1):19-73.

8. Foucar E, Rosai J, Dorfman RF, Eyman JM. Immunologic abnormalities and their significance in sinus histiocytosis with massive lymphadenopathy. Am J Clin Pathol 1984;82(5):515-525.

9. Kare M, Dang S, Dang A. Rosai-Dorfman syndrome with sinonasal mucosa and intraocular involvement. J Assoc Physicians India 2007;55:448-450.

10. Fudenberg HH, Good RA, Hitzig W, et al. Classification of the primary immune deficiencies: WHO recommendation. N Engl J Med 1970;283(12): 656-657. http://dx.doi.org/10.1056/NEJM197009172831211

11. Stebbing C, van der Walt J, Ramadan G, Inusa B. Rosai-Dorfman disease: A previously unreported association with sickle cell disease. BMC Clin Pathol 2007;7:3. http://dx.doi.org/10.1186/1472-6890-7-3

12. Ambati S, Chamyan G, Restrepo R, et al. Rosai-Dorfman disease following bone marrow transplantation for pre-B cell acute lymphoblastic leukemia. Pediatr Blood Cancer 2008;51(3):433-435. http://dx.doi.org/10.1002/pbc.21606

13. Odinaka KK, Nwolisa EC. Challenges in the management of the child with Duchenne muscular dystrophy in a resource poor setting: A case report. Pan Afr Med J 2014;19:227. http://dx.doi.org/10.11604/pamj.2014.19.227.3137

Accepted 12 August 2016 